\title{
Characteristics of Cat Skeletal Muscles Grafted with Intact Nerves or with Anastomosed Nerves
}

\author{
John A. FAUlKNer, John M. MARKLEY, JR., KeVin K. MCCully, \\ Christopher R. WATTERS, AND TIMOTHY P. White ${ }^{1}$
}

\begin{abstract}
Departments of Physiology, Plastic and Reconstructive Surgery, and Kinesiology, The University of Michigan. Ann Arbor, Michigan 48109
\end{abstract}

Received October 15, 1982; revision received January 10, 1983

\begin{abstract}
Grafting of 3-g extensor digitorum longus (EDL) muscles of cats may be made with (i) severence of the nerve with spontaneous reinnervation, termed standard grafts (ii) severence of the nerve with reinnervation facilitated by anastomosis of the nerve, termed nerve-anastomosed grafts; and (iii) preservation of the nerve, termed nerveintact grafts. In previous studies, standard grafts developed a maximum isometric tetanic tension $\left(\mathrm{P}_{0}\right)$ that was $22 \%$ of the value for control EDL muscles. We hypothesized that the low values of $P_{0}$ resulted from incomplete reinnervation of muscle fibers. To test this hypothesis, EDL muscles were grafted in cats with nerves intact and with nerves anastomosed. In standard grafts differences were observed in both structure and function at 120 compared with 240 days after grafting. Characteristics of the nerve-intact and nerve-anastomosed grafts did not change significantly between 120 and 240 days and the data were pooled for comparisons with control EDL muscles. Nerve-anastomosed and nerve-intact grafts developed $P_{0}$ values that were 34 and $64 \%$ of the control values, respectively. Nerve-intact grafts had a mass and fiber crosssectional area not different from control EDL muscles. Compared with control values, all grafts had fewer fibers, more connective tissue, lower absolute and normalized $\mathbf{P}_{0}$, reduced capillary density, and increased fatigability. The greater $P_{0}$ of nerve-intact compared with standard and nerve-anastomosed grafts supported our hypothesis that the degree of reinnervation is a factor that limits graft development. The presence of a necrotic core and the low tension development of even the nerve-intact grafts suggested that revascularization is a significant limitation as well.
\end{abstract}

Abbreviations: EDL-extensor digitorum longus, $\mathrm{P}_{\mathbf{0}}$-maximum isometric tetanic tension, TPT-time to peak twitch tension, ChAc-choline acetyltransferase, SDH-succinic acid dehydrogenase.

${ }^{1}$ Carol Brangwyn assisted with the histochemical incubations and data reduction, and Casey Donovan, Steven Segal, and John Villanacci with the biochemical assays. The study was supported by National Institutes of Health grants NS 17017 and AM 29732. 


\section{INTRODUCTION}

Successful restoration of function has been reported for free whole skeletal muscle autografts in rats (3), cats (10), monkeys $(18,20)$, and humans (12). With standard grafting procedures, tendons of the free grafts were sutured to the proximal and distal tendon stumps. Blood vessels and nerves were severed but not anastomosed. Consequently, revascularization (13) and reinnervation (5) occurred spontaneously. The ischemia that follows grafting resulted in degeneration of all but a few fibers $(4,23)$. Regeneration of skeletal muscle fibers was evident in those grafts but compared with values for control muscles, the maximum isometric tetanic tension $\left(\mathrm{P}_{0}\right)$ developed by grafts in rats was $57 \%$ (3) and in cats $22 \%$ (10). Standard grafts stabilized between 60 and 90 days in rats $(3,4)$ and between 150 and 380 days in cats $(10)$.

When the nerve to a muscle was left intact at the time of grafting, the subsequent ischemia of the muscle resulted in rapid degeneration of the intramuscular portions of the nerve fibers (4). The degree to which persisting elements of nerves, such as the basal lamina or the perineurial sheaths, provide conduits for reinnervation is not known. Grafting of muscles in rats with no surgical disruption of the motor nerve (nerve-intact grafts) resulted in a more rapid and complete recovery of structure and function. The $\mathbf{P}_{0}$ of the nerve-intact grafts averaged $91 \%$ of the control value (4).

The development of the nerve-intact model for grafting small muscles in rats enabled us to test four hypotheses in large muscle grafts in cats: (i) Cumpared with standard grafts, grafts made with nerves intact or nerves anastomosed would achieve a stabilized condition of structure and function more rapidly; (ii) grafts made with the nerves intact or nerves anastomosed would have a greater restoration of structure and function than standard grafts; (iii) grafts made with the nerves intact would not be significantly different in structure and function from grafts transplanted with nerves anastomosed; and (iv) all grafts would have some impairment in structure and function compared with control values.

\section{METHODS}

Data were obtained on adult male or female cats 1.7 to $6.2 \mathrm{~kg}$ in body mass $(\bar{x} \pm \mathrm{SE}=3.4 \pm 0.2 \mathrm{~kg})$. Control data were obtained on six extensor digitorum longus (EDL) muscles. The data were compared with our published data on control EDL muscles of cats $(10,21)$. Because no significant differences were observed, the data were pooled. The 23 cats in which skeletal muscles were grafted were healthy and free from disease. For the grafting of muscles and collection of data, the cats were anesthetized by an i.m. injection of ketamine hydrochloride $(10 \mathrm{mg} / \mathrm{kg})$ followed by an i.v. injection of sodium pentobarbital $(10 \mathrm{mg} / \mathrm{kg})$. Supplemental injections of sodium pentobarbital 
were made as required. After completion of data collection the animals were killed with an overdose of pentobarbital.

The EDL muscles were grafted back into the same site from which they were removed. We have shown $(7,10,21)$ that those grafts (orthotopic grafts) are not significantly different from EDL muscles grafted into the contralateral limb (heterotopic grafts). Grafting was in both hind limbs of each cat. Each EDL muscle was grafted by cutting all vascular connections and the proximal and distal tendons. The muscle was removed from its normal site and replaced in its original orientation. The proximal and distal tendons were sutured to the tendon stumps. All grafts were ischemic until revascularization occurred spontaneously. The three types of grafts differed as to the operative procedure used on the motor nerve. For standard grafts $(N=18)$, the nerves were transected at the time of grafting and reinnervation occurred spontaneously (10). Grafts with anastomosis of the motor nerve $(N=9)$ were obtained by cutting the nerves, and then the cut end of the peroneal nerve was sutured to the stump of the nerve pedicle of the graft (20). A nerveintact graft $(N=13)$ was made with no disruption of the major motor nerves (4) but the small blood vessels associated with the nerves were electrocoagulated.

Animals chosen randomly were anesthetized at 120 or 240 days after grafting for the collection of data on the structure and function of the grafts. The same data were collected on control EDL muscles. For the measurement of contractile properties, the graft was freed with proximal tendon, nerves, and blood vessels intact. The distal tendon of the EDL graft was attached with a hook to a modified Fales muscle lever. Contractions were elicited by direct stimulation of the whole muscle with supramaximal square wave pulses. Measurements were made during isometric contractions with the muscle fibers at optimal length for maximal twitch tension development by methods described by us previously (10).

Muscles and grafts were stimulated directly because the diffuse innervation of standard grafts through original channels and sprouts made indirect stimulation through the nerve difficult to achieve and interpret. Some small nerves were damaged while dissecting the standard grafts and others could not be stimulated. Furthermore, for eight control EDL muscles and six nerve-intact grafts, no significant differences in contractile properties were observed between direct and indirect stimulation. We concluded that a comparison of $\mathrm{P}_{0}$ s obtained by direct and indirect stimulation would not provide a discriminating test for our experiments.

Measurements of contractile properties included time to peak twitch tension (TPT); one-half relaxation time, maximum twitch tension, maximum isometric tetanic tension $\left(\mathrm{P}_{0}\right)$, twitch tension:tetanic tension ratio, and fre- 
quency:force relationship at stimulation frequencies of $10,20,35,50,80$, and $120 \mathrm{~Hz}$. The $P_{0}$ was normalized per square centimeter of cross-sectional area based on mass and fiber length (10). The maximum velocity of shortening was determined from the velocity of shortenings at 11 to 15 afterloads between 0.02 and 0.80 of $P_{0}$ and was calculated from the linear form of the Hill Equation (14). The fatigability of grafts and EDL muscles was assessed by stimulation once per second with 0.2 -ms pulses at $70 \mathrm{~Hz}$ for $200 \mathrm{~ms}$. The fatigue index was the time required for tetanic tension to decrease to $50 \%$ of the initial value.

After measurement of contractile properties, the graft or control muscle was removed and weighed. A bundle of intact skeletal muscle fibers was removed for measurement of choline acetyltransferase (ChAc) activity. The ChAc activity, which provides an estimate of the mass of cholinergic nerve terminals (4-6), was used as a quantitative assessment of the degree of reinnervation in the different grafts. Because multiple bundles of intact fibers from the same muscle demonstrated no significant differences in ChAc activity, bundles were used to estimate ChAc activity of whole muscles. That activity was measured by Tǔcek's (24) modification of Fonnum's radiometric method.

The pennate nature of the EDL muscles and the EDL grafts enabled us to obtain a complete cross section through the belly of the graft or muscle just below the end of the longitudinal sample. This sample was quick-frozen in isopentane and dry ice, and cross sections of $10-\mu \mathrm{m}$ thickness were cut and subsequently incubated for succinic acid dehydrogenase activity, myofibrillar ATPase activity, and capillary membrane phosphatase activity (19, 21,22 ). Serial sections of each graft or control EDL muscle wcre projectcd at $1000 \times$ magnification and four to six sites were sampled randomly from the periphery and two to four from the center one-third of each cross section. The average number of fibers sampled at each site was 35 (range 15 to 90 due to variation in fiber area and amount of connective tissue). Fiber classification was based on type I fibers having low and type II fibers having high myofibrillar ATPase activities (Fig. 3A). In serial sections, succinic acid dehydrogenase (SDH) activity (Fig. 2B, D) was used to identify type II fibers that were high (type IIA) and low in oxidative capacity (type IIB). The remainder of the tissue was homogenized in $175 \mathrm{mM} \mathrm{KCl}$ and $2 \mathrm{mM}$ EDTA,

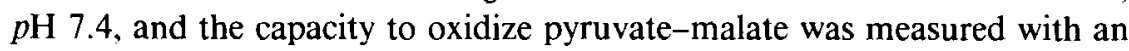
oxygen electrode at $30^{\circ} \mathrm{C}(15)$.

Data are presented as the mean \pm 1 standard error. Statistical significance of differences between means was determined by $t$ tests $(P<0.05)$ using the Welch-Aspin convention which does not require equal sample sizes and assumes variances to be unknown and not necessarily equal (17). 


\section{RESULTS}

Comparisons made between 120 and 240 days after grafting showed no significant differences for any variable for either nerve-intact or nerve-anastomosed grafts. For standard grafts at 120 days compared with 240 days, the TPT was prolonged and both the absolute and normalized $\mathrm{P}_{0} \mathrm{~s}$ were lower (Table 1). Comparisons at 120 days among the three types of grafts indicated that grafts with nerve repair had a more rapid recovery toward control values than standard grafts for TPT, absolute $P_{0}$, and normalized $P_{0}$ (Table 1). Nerve-intact grafts also had a significantly larger muscle mass than standard grafts. Compared with standard grafts at 240 days, nerve-anastomosed grafts showed few significant differences whereas nerve-intact grafts showed significantly greater improvement for almost all variables (Table 1).

Because no significant difference was observed between 120 and 240 days for any characteristic of nerve-intact or nerve anastomosed grafts, the data for each type of graft at the two periods were pooled. Comparisons of the

\section{TABLE 1}

Structural and Functional Variables of Standard, Nerve-Anastomosed, and Nerve-Intact Grafts 120 and 240 Days after Grafting ${ }^{a}$

\begin{tabular}{|c|c|c|c|c|c|c|}
\hline & \multicolumn{2}{|c|}{ Standard grafts } & \multicolumn{2}{|c|}{ Nerve-anastomosed grafts } & \multicolumn{2}{|c|}{ Nerve-intact grafts } \\
\hline & $\begin{array}{l}120 \text { days } \\
(N=8)\end{array}$ & $\begin{array}{l}240 \text { days } \\
(N=10)\end{array}$ & $\begin{array}{l}120 \text { days } \\
(N=4)\end{array}$ & $\begin{array}{l}240 \text { days } \\
(N=5)\end{array}$ & $\begin{array}{l}120 \text { days } \\
(N=8)\end{array}$ & $\begin{array}{c}240 \text { days } \\
(N=5)\end{array}$ \\
\hline Muscle mass (g) & $1.9 \pm 0.15$ & $1.8 \pm 0.29$ & $2.2 \pm 0.3$ & $1.5 \pm 0.4$ & $3.2 \pm 0.4 \uparrow$ & $3.2 \pm 0.2 \dagger$ \\
\hline $\begin{array}{l}\text { Cross-sectional } \\
\text { area of } \\
\text { muscle }\left(\mathrm{cm}^{2}\right)\end{array}$ & $0.67 \pm 0.04$ & $0.53 \pm 0.05^{*}$ & $0.63 \pm 0.10$ & $0.47 \pm 0.12$ & $0.84 \pm 0.09$ & $0.86 \pm 0.12$ \\
\hline $\begin{array}{l}\text { Time to peak } \\
\text { twitch tension } \\
\text { (ms) }\end{array}$ & $44.1 \pm 4.9$ & $30.3 \pm 1.5^{*}$ & $25.5 \pm 1.3 \dagger$ & $30.0 \pm 3.4$ & $25.1 \pm 0.9 \dagger$ & $23.5 \pm 1.7 \dagger$ \\
\hline $\begin{array}{l}\text { Maximum } \\
\text { isometric } \\
\text { tetanic } \\
\text { tension }(\mathrm{N})\end{array}$ & $2.57 \pm 0.37$ & $7.85 \pm 1.80^{*}$ & $8.9 \pm 1.1 \dagger$ & $8.4 \pm 2.3$ & $15.3 \pm 0.6 \dagger$ & $18.2 \pm 1.4 \dagger$ \\
\hline $\begin{array}{l}\text { Maximum } \\
\text { isometric } \\
\text { tetanic } \\
\text { tension }(\mathrm{N} / \\
\left.\mathrm{cm}^{2}\right)\end{array}$ & $3.9 \pm 0.6$ & $13.7 \pm 2.6^{*}$ & $14.6 \pm 1.7 \dagger$ & & & \\
\hline Fatigue index (s) & $57 \pm 11$ & $78 \pm 9$ & $68 \pm 4$ & $72 \pm 6$ & $60 \pm 4$ & $69 \pm 6$ \\
\hline Fiber area $\left(\mu \mathrm{m}^{2}\right)$ & $2093 \pm 215$ & $2901 \pm 273^{*}$ & $2046 \pm 255$ & $2164 \pm 231$ & $2894 \pm 606$ & $2787 \pm 804$ \\
\hline
\end{tabular}

${ }^{a}$ Data are $\bar{X} \pm$ SE. Significant differences $(P<0.05)$ between 240 and 120 days for the same type of graft are indicated by ${ }^{*}$, and between nerve-anastomosed or nerve-intact grafts and standard grafts at the same time period by + . 
pooled data with data for control EDL muscles indicated that nerve-intact and nerve-anastmosed grafts had prolonged TPTs, decreased absolute and normalized $\mathrm{P}_{0} \mathrm{~s}$, increased fatigabilities (Table 2), decreased percentages of type I fibers, increased percentages of type IIB fibers, and decreased capillary densities per square millimeter and per fiber (Table 3). Compared with control values, the grafts made with anastomosis of the nerve also had a decreased cross-sectional area, a prolonged one-half relaxation time, and a decreased capacity for pyruvate-malate oxidation. Significant differences for the nerveanastomosed grafts compared with nerve-intact grafts were a smaller muscle mass and muscle cross-sectional area, and a smaller absolute $\mathrm{P}_{0}$ (Table 2).

The frequency-force curves for nerve-intact and nerve-anastomosed grafts at 120 and 240 days and for standard grafts at 240 days were not significantly different from the control values (Fig. 1). For any given frequency, the relative force developed by 120 -day standard grafts was significantly greater until the $P_{0}$ was attained. Those observations agreed with our previous observations of standard grafts (10).

Photomicrographs indicated that in spite of greater restoration of function in nerve-intact grafts, a necrotic core was still observed in some grafts (Fig.

TABLE 2

The Contractile Properties and Related Morphologic Variables of Control Extensor Digitorum Longus (EDL) Muscles and Stabilized Nerve-Anastomosed and Nerve-Intact Grafts ${ }^{a}$

\begin{tabular}{|c|c|c|c|}
\hline & $\begin{array}{c}\text { Control EDL } \\
\text { muscles }(N=12)\end{array}$ & $\begin{array}{l}\text { Nerve-anastomosed } \\
\text { grafts }(N=9)\end{array}$ & $\begin{array}{c}\text { Nerve intact } \\
\text { grafts }(N=13)\end{array}$ \\
\hline Muscle mass (g) & $2.82 \pm 0.18$ & $1.87 \pm 0.25^{*}$ & $3.22 \pm 0.32 \dagger$ \\
\hline Fiber length (mm) & $28.4 \pm 0.6$ & $27.5 \pm 1.7$ & $29.9 \pm 1.0$ \\
\hline $\begin{array}{l}\text { Cross-sectional area of muscle } \\
\left(\mathrm{cm}^{2}\right)\end{array}$ & $0.92 \pm 0.03$ & $0.55 \pm 0.08^{*}$ & $0.85 \pm 0.08 \dagger$ \\
\hline $\begin{array}{l}\text { Time to peak twitch tension } \\
\text { (ms) }\end{array}$ & $19.2 \pm 0.60$ & $27.8 \pm 2.30^{*}$ & $24.5 \pm 0.80^{*}$ \\
\hline One-half relaxation time (ms) & $20.5 \pm 1.30$ & $26.1 \pm 2.00^{*}$ & $23.8 \pm 1.80$ \\
\hline Twitch:tetanus ratio & $0.18 \pm 0.01$ & $0.17 \pm 0.02$ & $0.17 \pm 0.01$ \\
\hline Maximum tetanic tensions $(\mathrm{N})$ & $25.2 \pm 1.0$ & $8.5 \pm 1.2^{*}$ & $16.0 \pm 1.8^{*} \dagger$ \\
\hline $\begin{array}{l}\text { Maximum tetanic tension }(\mathrm{N} / \\
\left.\mathrm{cm}^{2}\right)\end{array}$ & $27.5 \pm 1.1$ & $16.4 \pm 1.1^{*}$ & $18.6 \pm 2.2^{*}$ \\
\hline $\begin{array}{l}\text { Maximum velocity of shorten- } \\
\text { ing }\left(\mathrm{L}_{0} / \mathrm{s}\right) \\
\text { Fatigue index (s) }\end{array}$ & $\begin{array}{l}13.5 \pm 1.2 \\
165 \pm 5\end{array}$ & $\begin{aligned} 10.9 & \pm 3.6 \\
70 & \pm 4^{*}\end{aligned}$ & $\begin{aligned} 11.7 & \pm 1.2 \\
64 & \pm 3^{*}\end{aligned}$ \\
\hline
\end{tabular}

${ }^{a}$ Data are $\bar{x} \pm \mathrm{SE}$. Significant differences $(P<0.05)$ between nerve-intact or nerve-anastomosed grafts and control EDL muscles are indicated by ${ }^{*}$ and between nerve-anatomosed and nerveintact grafts by $\dagger$. 
TABLE 3

Morphologic Characteristics of Control EDL Muscles, Stabilized Nerve-Anastomosed Grafts, and Stabilized Nerve-Intact Grafts ${ }^{a}$

\begin{tabular}{|c|c|c|c|}
\hline & $\begin{array}{c}\text { Control EDL } \\
\text { muscles }(N=12)\end{array}$ & $\begin{array}{l}\text { Nerve-anastomosed } \\
\text { grafts }(N=9)\end{array}$ & $\begin{array}{c}\text { Nerve-intact } \\
\text { grafts }(N=13)\end{array}$ \\
\hline \multicolumn{4}{|l|}{ Area composition } \\
\hline Type I (\%) & $14 \pm 1$ & $5 \pm 1^{*}$ & $4 \pm 2^{*}$ \\
\hline Type IIA (\%) & $35 \pm 4$ & $25 \pm 6$ & $19 \pm 5^{*}$ \\
\hline Type IIB (\%) & $51 \pm 5$ & $70 \pm 7^{*}$ & $77 \pm 6^{*}$ \\
\hline $\begin{array}{l}\text { Cross-sectional area of } \\
\text { fibers }\left(\mu \mathrm{m}^{2}\right)\end{array}$ & $2308 \pm 159$ & $2104 \pm 160$ & $2840 \pm 451$ \\
\hline $\begin{array}{l}\text { Pyruvate-malate oxidation } \\
\qquad\left(\mu \mathrm{l} \mathrm{O}_{2} \cdot h^{-1} \cdot \mathrm{mg}_{\text {protein }}{ }^{-1}\right)\end{array}$ & $5.77 \pm 0.62$ & $1.32 \pm 0.32^{*}$ & $3.71 \pm 1.20$ \\
\hline $\begin{array}{l}\text { Choline acetyltransferase } \\
\left(\mathrm{pM} \mathrm{ChAc} \cdot \mathrm{h}^{-1} \cdot \mathrm{mg}\right. \\
\left.\operatorname{protein}^{-1}\right)\end{array}$ & $81.2 \pm 5.8$ & $66.1 \pm 10.6$ & $69.3 \pm 8.9$ \\
\hline Capillaries $/ \mathrm{mm}^{2}$ & $624 \pm 27$ & $323 \pm 20^{*}$ & $286 \pm 38^{*}$ \\
\hline Capillaries/fiber ratio & $1.68 \pm 0.08$ & $0.91 \pm 0.08^{*}$ & $0.97 \pm 0.10^{*}$ \\
\hline
\end{tabular}

${ }^{a}$ Data are $\bar{x} \pm$ SE. Significant differences $(P<0.05)$ between nerve-intact or nerve anastomosed grafts and control EDL muscles are indicated by *. Among these variables, no significant differences were observed between nerve-intact and nerve-anastomosed grafts.

2). In addition to the regeneration of fewer type I fibers, those fibers in some grafts tended to cluster in groups of two to eight (Fig. 3C, D) rather than single fibers being broadly dispersed among the type II fibers (Fig. 3A, B).

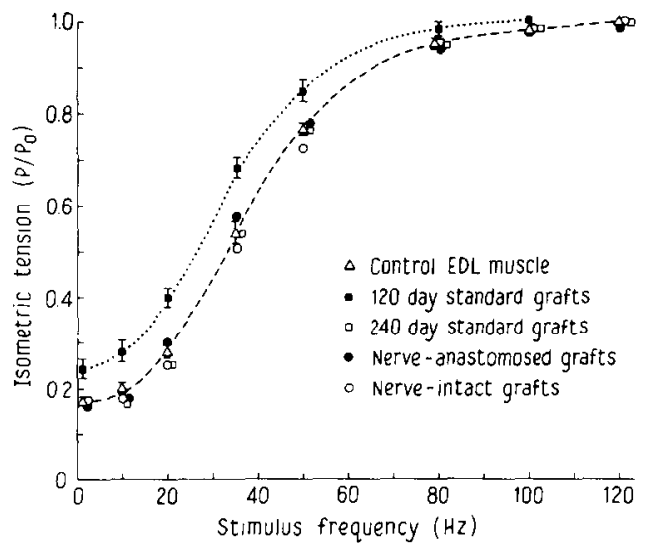

Fig. 1. The frequency-force relationships for standard grafts at 120 and 240 days after transplantation, pooled data on nerve-anastomosed and nerve-intact grafts, and data on control extensor digitorum longus (EDL) muscles. The $P / P_{0}$ is the ratio of the isometric tension developed at a given stimulation frequency $(P)$ and the maximum isometric tetanic tension $\left(\mathrm{P}_{0}\right)$. 


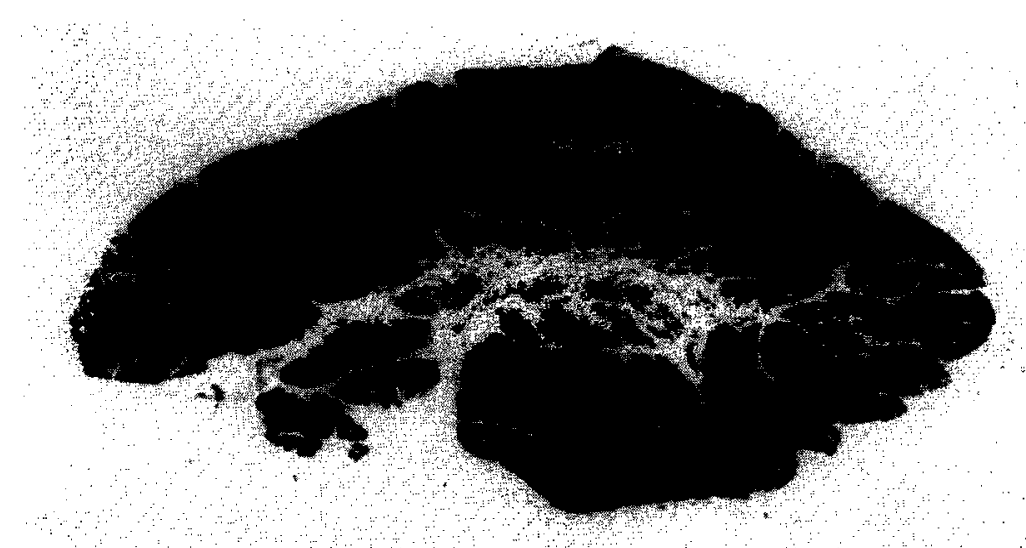

FiG. 2. Cross section of a nerve-intact graft of EDL muscle 120 days after orthotopic grafting. The section was incubated for myofibrillar ATPase activity. Magnification $\times 8.6$.

\section{DISCUSSION}

In each of the three types of grafts, sections removed 3 to 7 days after grafting showed degenerative changes in approximately $98 \%$ of the skeletal muscle fibers. Surviving fibers were situated in the periphery of the grafts. Regeneration of fibers proceeded from the periphery to the center. Those observations are consistent with previous data on standard grafts in cats (23), nerve-anastomosed grafts in monkeys (20), and standard and nerve-intact grafts in rats (4).

We have defined a stabilized graft as a graft in which the mean fiber crosssectional area in the central portion of the graft did not differ significantly from that in the peripheral portion $(7,9)$. By that criterion, standard grafts required 150 to 380 days to become stabilized (10). Our study indicates that this criterion is not satisfactory. Some grafts showed no difference in fiber cross-sectional area in the center compared with the periphery at 120 or 240 days. Other grafts had significantly larger fibers in the periphery than in the center (Table 4). In spite of variations in the mean fiber area between the center and the periphery, the mean fiber area of the total graft, the mean cross-sectional area of the total graft, and the $P_{0}$ remained constant between 120 and 240 days. The nerve-intact and nerve-anastomosed grafts stabilized functionally by 120 days despite regional differences in fiber area. We have proposed the absolute $P_{0}$ as the best single measure of the restoration of function for a graft $(7,10)$. We now advocate no change in $P_{0}$ with time as the best criterion of a graft stabilized as to function. 

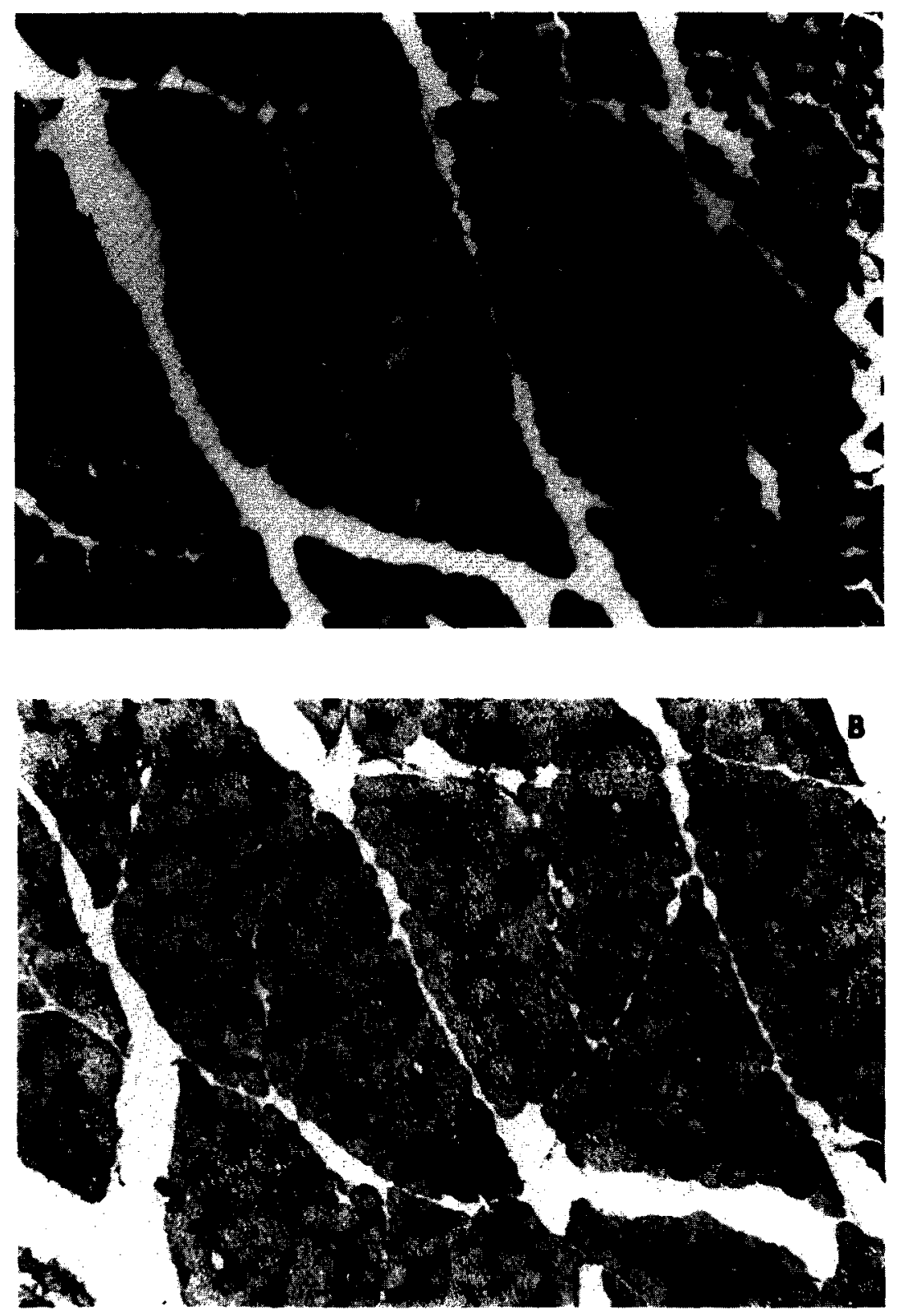

FIG. 3. Photomicrographs of serial sections of control (A, B) and a 120-day nerve-intact graft (C, D) incubated for myofibrillar ATPase $(A, C)$ and for succinic acid dehydrogenase activity (B, D). Note that in control EDL muscles type I fibers are dispersed widely whereas in nerveintact graft the type I fibers are clustered. Magnification $\times 38.25$. 

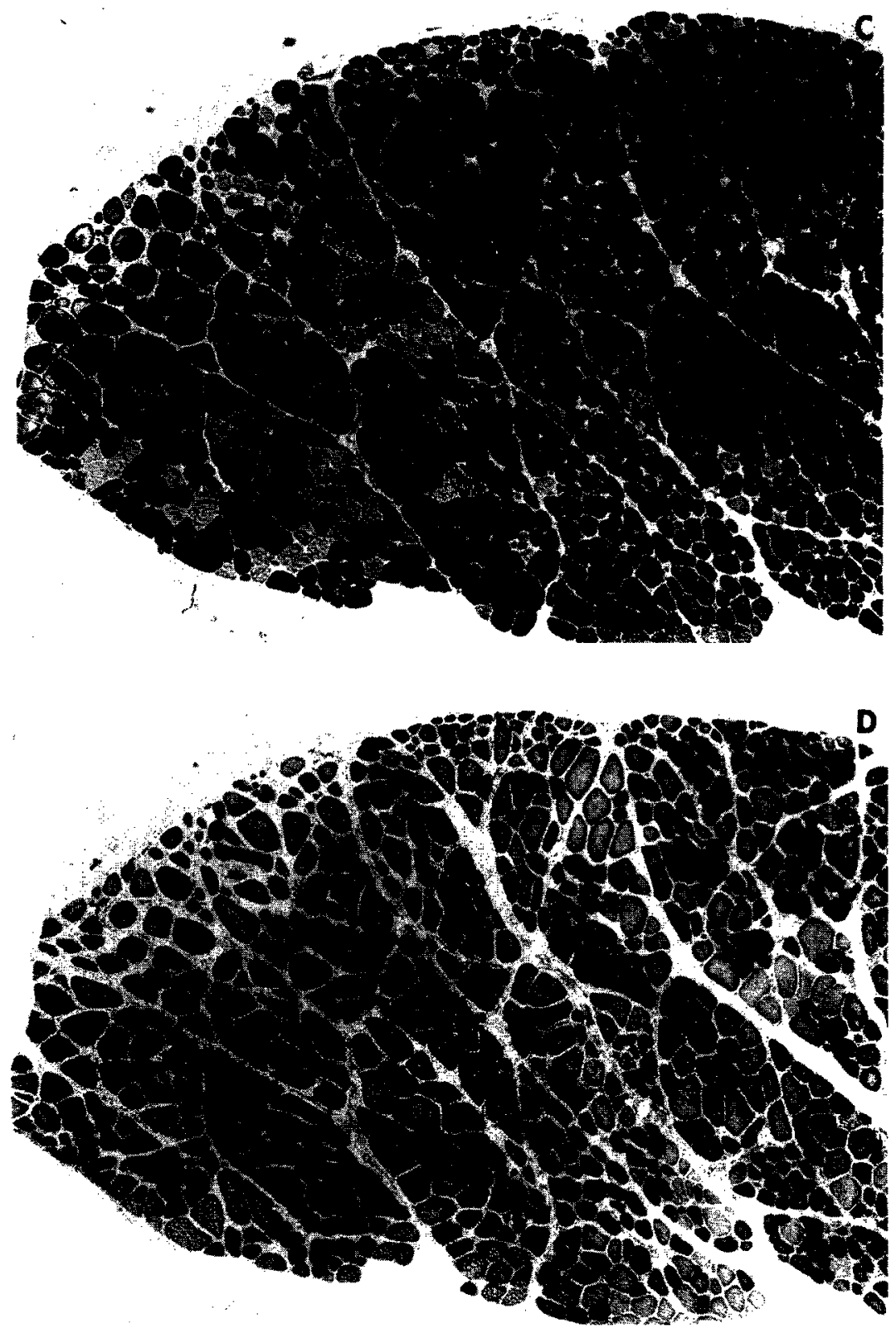

FIG. 3-Continued. 
TABLE 4

Cross-Sectional Areas of Skeletal Muscle Fibers from the Central and Peripheral Sites of Selected Nerve-Anastomosed and Nerve-Intact Grafts and for the Total Groups ${ }^{a}$

\begin{tabular}{|c|c|c|c|c|c|}
\hline \multicolumn{3}{|c|}{120 days } & \multicolumn{3}{|c|}{240 days } \\
\hline Graft & Central & Peripheral & Graft & Central & Peripheral \\
\hline \multicolumn{6}{|c|}{ Nerve-anastomosed grafts } \\
\hline a & $899 \pm 88$ & $2712 \pm 161$ & $\mathrm{c}$ & $1007 \pm 59$ & $3878 \pm 186$ \\
\hline b & $2384 \pm 218$ & $2817 \pm 126$ & d & $2764 \pm 155$ & $2625 \pm 137$ \\
\hline $\bar{X}(N=4)$ & $1522 \pm 312$ & $2631 \pm 141$ & $\bar{X}(N=5)$ & $1819 \pm 421$ & $2696 \pm 422$ \\
\hline \multicolumn{6}{|c|}{ Nerve-intact grafts } \\
\hline a & $920 \pm 114$ & $5433 \pm 462$ & c & $2120 \pm 143$ & $3152 \pm 119$ \\
\hline $\mathrm{b}$ & $1611 \pm 138$ & $2401 \pm 156$ & d & $4359 \pm 355$ & $4137 \pm 175$ \\
\hline $\bar{X}(N=8)$ & $1963 \pm 513$ & $4187 \pm 647$ & $\bar{X}(N=5)$ & $2586 \pm 650$ & $2918 \pm 551$ \\
\hline
\end{tabular}

${ }^{a}$ Grafts a to $d$ are $\vec{X} \pm$ SE for single grafts. The number of fibers sampled for a given graft was $92 \pm 16$ fibers for central sites and $144 \pm 16$ for peripheral sites. The mean of the $\bar{x} \pm \mathrm{SE}$ is given for the total number of grafts in each group. The number of grafts is in parentheses.

Compared with standard grafts the more rapid restoration and stabilization of $\mathrm{P}_{0}$ in grafts made with nerves anastomosed or with nerves intact supports our hypothesis that reinnervation of standard grafts was both slow and incomplete. A major concern of previous studies of grafts in cats (10), and monkeys (20) was the attainment by standard grafts of only 22 to $29 \%$ of the control values for $P_{0}$. In our study, the standard grafts were slightly better, with a $\mathrm{P}_{0} 31 \%$ of the control value. The $\mathrm{P}_{0}$ of the nerve-anastomosed grafts of $34 \%$ of the control value was not different from standard grafts but the nerve-intact grafts achieved $64 \%$. We conclude that the operative technique for nerve anastomosis was not as effective as the nerve-intact procedure for the restoration of $\mathrm{P}_{0}$.

The $P_{0}$ of the nerve-intact grafts in cats relative to values for control EDL muscles was significantly less than the $91 \%$ reported for nerve-intact EDL grafts of rats (4). The difference is likely attributable to the larger mass of muscle grafted, 3 to $6 \mathrm{~g}$ in cats compared with $100 \mathrm{mg}$ in the rat, and is not a difference due to species. A necrotic core which was observed in all types of grafts in cats (Fig. 2) and grafts of similar mass in monkeys (20), was never seen in grafts in rats (4). The necrotic core likely resulted from inadequate revascularization of the central portion of large grafts. The presence of the necrotic core resulted in fewer muscle fibers per cross-sectional area and a lower $\mathrm{P}_{0}$ compared with control values. 
Based on mass, we estimated the number of fibers in control EDL muscles and standard grafts as 45,000 and 13,000 , respectively $(7,9)$. When corrected for connective tissue and the necrotic core, grafts, as well as control EDL muscles, developed a $P_{0}$ of $28 \mathrm{~N} / \mathrm{cm}^{2}(10)$. Thus, an estimate of the functional cross-sectional area $\left(\mathrm{cm}^{2}\right)$ that was developing tension was obtained by dividing the absolute $P_{0}$ in Newtons by $28 \mathrm{~N} / \mathrm{cm}^{2}$. The functional cross-sectional area divided by the mean fiber area then provided an estimate of the number of fibers per graft or muscle. Those calculations yielded estimates of 39,000 fibers for control EDL muscles, 9,000 for standard grafts, 15,000 for nerve-anastomosed grafts, and 20,000 for nerve-intact grafts. The estimates for fiber number for control EDL muscles and for standard grafts were slightly lower than our previous estimates $(7,9)$ but the proportionality between standard grafts and control EDL muscles was the same. Compared with the standard grafting procedure, nerve-intact and nerve-anastomosed grafting procedures enhanced the number of fibers that regenerated.

Carlson et al. (4) found significant differences for total muscle ChAc activity among standard grafts, nerve-intact grafts, and control EDL muscles of rats. The ChAc activity of $46.4 \pm 7.5 \mathrm{pmol} \mathrm{ChAc} \cdot \mathrm{h}^{-1} \cdot \mathrm{mg}$ protein ${ }^{1}$ for standard grafts in cats was significantly less than the value for control EDL muscles, for nerve-anastomosed grafts, or for nerve-intact grafts. The lack of significant differences between the nerve-anastomosed and nerve-intact grafts and control values appeared to be due to an increased variability resulting from sampling problems in the relatively large cat EDL muscles.

Type-grouping is the clustering together of fibers of the same histochemical type in much larger groups than usual (16) and is one sign of neuronal regeneration. After either nerve crush or nerve anastomosis, type-grouping results from a reduction in the number of nerve fibers that reach the muscle and reinnervation of additional fibers by collateral sprouting (16). We reported type-grouping previously in standard EDL grafts of cats (8). In the present study, standard EDL grafts either had no type I fibers or type-grouping was observed. Whereas $56 \%$ of the nerve-anastomosed grafts and $23 \%$ of the nerve-intact grafts showed type grouping. The degree to which type-grouping was present appeared to be inversely related to the success with which skeletal muscle fibers in a motor unit were reinnervated in a manner comparable to the control EDL muscle. Because grouping of type I fibers was observed equally at 120 and 240 days, the phenomenon appeared fixed and was not modified during this time.

The capillary density of nerve-anastomosed and nerve-intact grafts was similar to that reported previously for standard grafts $(20,21)$. All three types of grafts had approximately one-half the capillary density of control EDL muscles. The low capillary density was associated with a rapid fatigability of 
each type of graft. In a number of different control muscles, no significant relationship was observed between capillary density and fatigability but the values between 280 to 320 capillaries per square millimeter for grafts were below the range represented $(19,22)$. The maximum nutrient blood flow estimated per unit mass of viable fibers of standard grafts was $170 \%$ of control values (25). The high flow coupled with the low capillary density implies a capillary red blood cell velocity in grafts almost fourfold greater than in control EDL muscles. The fast transit time and the long diffusion distances might have contributed to the rapid onset of fatigue for grafts.

Standard and nerve-anastomosed grafts had low pyruvate-malate oxidation which was consistent with their rapid fatigability. The rapid fatigability of the nerve-intact grafts occurred in spite of high pyruvate-malate oxidation. The high SDH activity for many type I and type IIA fibers observed in photomicrographs of nerve-anastomosed and nerve-intact grafts suggested that some of the individual fibers or motor units might be quite resistant to fatigue. The rapid loss of tension by grafts when the whole graft was stimulated with supramaximal stimuli likely resulted from the higher percentage of type IIB fibers present in all types of grafts compared with control EDL muscles. It is well documented that type IIB fibers or motor units are highly fatigable (1).

Our study of nerve-intact and nerve-anastomosed grafts in cats supports a significant role for nerve repair in the improvement of the number and the functional mass of skeletal muscle fibers that regenerate after whole muscle grafting. Compared with standard grafts, anastomosis or preservation of the motor nerve resulted in more rapid stabilization of grafts and improved reinnervation. Greater mass and tension development for the nerve-intact than for nerve-anastomosed grafts indicated that microsurgical techniques for nerve anastomosis were not optimal. In a comparison of microsurgical techniques for nerve repair of the vascularized rectus femoris muscle of rabbits, Frey et al. (11) reported a $\mathrm{P}_{0}$ for muscles with nerve anastomosis that was $75 \%$ of the control value. That contrasts with our value of $34 \%$ for nerveanastomosed grafts. Grafting with nerves intact resulted in a restoration of $64 \%$ of the $\mathrm{P}_{0}$ of control EDL muscles, which suggests that an interaction between the timing of the reinnervation and regenerative processes in nerveanastomosed grafts may be critical. Carlson et al. (2) reported that the difference in the timing of restoration of neuromuscular function between nerveintact ( 8 days) and standard ( 21 to 23 days) grafts was not the critical factor accounting for the relative success of nerve-intact grafts. However, the timing of reinnervation might be of importance if denervation was extended for longer periods. Reinnervation of fibers in EDL grafts in cats requires as much as 40 days. The presence of a necrotic core and a decreased $P_{0}$ in all three 
types of grafts indicates that adequate revascularization continues to constitute a limitation. Much remains to be understood of the roles of reinnervation and revascularization in the restoration of the structure and function of autografts.

\section{REFERENCES}

1. Burke, R. E., D. N. Levine, F. E. Azjac III, P. Tsairis, and W. K. Engel. 1971. Mammalian motor units: physiological-histochemical correlates in three types of cat gastrocnemius. Science 174: 709-712.

2. Carlson, B. M., A. H. Foster, D. M. Bader, P. HNIK, AND R. VejSAdA. 1983. Restoration of full mass in nerve-intact muscle grafts after delayed reinnervation. Experientia 39: $171-172$.

3. CARLSON, B. M., AND E. GUTMANN. 1975. Regeneration in grafts of normal and denervated rat muscles: contractile properties. Pflügers Arch. 353: 215-225.

4. Carlson, B. M., P. HNiK, S. Túcek, R. Vejsada, D. Bader, AND J. A. Faulkner. 1981. Comparison between grafts with intact nerves and standard free grafts of the rat extensor digitorum longus muscle. Physiol. Bohemoslov. 30: 505-513.

5. Carlson, B. M., K. R. WAGNER, AND S. R. MAX. 1979. Reinnervation of rat extensor digitorum longus muscles after free grafting. Muscle Nerve 2: 304-307.

6. Diamond, I., G. M. Frankin, and D. Milfay. 1974. The relationship of choline acetyltransferase activity at the neuromuscular junction to changes in muscle mass and function. J. Physiol. (London) 236: 247-257.

7. FAULKNeR, J. A., J. M. MARKLEY, JR., AND T. P. White. 1981. Skeletal muscle transplantation in cats with and without nerve repair. Pages 47-54 in G. FreILINGER, J. HOLLE, AND B. M. CARLSON, Eds., Muscle Transplantation. Springer-Verlag, Vienna.

8. Faulkner, J. A., L. C. MaXwell, S. A. Mufti, and B. M. Carison. Skeletal muscle fiber regeneration following heterotopic autotransplantation in cats. Life Sci. 19: 289296, 1976.

9. Faulkner, J. A., L. C. MAXWell, T. P. White, AND J. H. Niemeyer. 1979. Characteristics of autografted mammalian skeletal muscles. Pages 485-492 in A. MAURO, Ed., Muscle Regeneration. Raven Press, New York.

10. Faulkner, J. A., J. H. Niemeyer, L. C. Maxwell, and T. P. White. 1980. Contractile properties of transplanted extensor digitorum longus muscles of cats. Am. J. Physiol. 238: C120-C126.

11. Frey, M., H. Gruber, J. Holle, AND G. Freilinger. 1982. An experimental comparison of the different kinds of muscle reinnervation: nerve suture, nerve implantation, and muscular neurotization. Plast. Reconstr. Surg. 69(4): 656-669.

12. Hakelius, L. 1974. Transplantation of free autogenous muscle in the treatment of facial paralysis. A clinical study. Scand. J. Plast. Reconstr. Surg. 8: 220-230.

13. HanSEN-Smith, F. M., B. M. CARLSON, AND K. L. IRWIN. 1980. Revascularization of the freely grafted extensor digitorum longus muscle in the rat. Am. $J$. Anat. 158: 65-82.

14. HILl, A. V. 1938. The heat of shortening and the dynamic constants of muscle. Proc. $R$. Soc. Lond. (Biol). 126: 136-195.

15. HoOKFr, A. M., AND K. M. Baldwin. 1979. Substrate oxidation specificity in different types of mammalian muscle. Am. J. Physiol. 236: C66-C69.

16. KarPatI, G., AND W. K. ENGEL. 1968. "Type grouping" in skeletal muscles after experimental reinnervation. Neurology 18: 447-455. 
17. Marascuilo, L. A. 1971. Statistical methods for behavioral science research. Pp. 312-314. McGraw, San Francisco.

18. MARKieY, J. M., JR., J. A. FAulkner, AND B. M. CARLson. 1978. Regeneration of skeletal muscle after grafting in monkeys. Plast. Reconstr. Surg. 62: 415-422.

19. MaXwell, L. C., J. K. BarClay, D. E. Mohrman, and J. A. Faulkner. 1977. Physiological characteristics of skeletal muscles of dogs and cats. Am. J. Physiol. 233: C14C18.

20. Maxwell, L. C., J. A. Faulkner, J. M. Markiey, JR., and D. R. Winborn. 1979. Neuroanastomosis of orthotopically transplanted palmaris longus muscles. Muscle Nerve 2: 44-52.

21. MaXwell, L. C., J. A. Faulkner, S. A. Mufti, and A. M. Turowski. 1978. Free autografting of entire limb muscles in the cat: histochemistry and biochemistry. $J$. Appl. Physiol. 44: 431-437.

22. Maxwell, L. C., T. P. White, AND J. A. FAULKNER. 1980. Oxidative capacity, blood flow, and capillarity of skeletal muscles. J. Appl. Physiol. 49: 627-633.

23. Mufti, S. A., B. M. Carlson, L. C. MaXwell, and J. A. Faulkner. 1977. The free grafting of entire limb muscles in the cat: morphology. Anat. Rec. 188: 417-430.

24. TŭCEK, S. 1978. Acetylcholine Synthesis in Neurons. Chapman and Hall, London.

25. White, T. P., L. C. MAXWEll, D. M. SOSIN, AND J. A. FAULKNER. 1981. Capillarity and blood flow of transplanted skeletal muscles of cats. Am. J. Physiol. 241: H630-H636. 